\title{
A CONJECTURE OF M. GOLOMB ON OPTIMAL AND NEARLY-OPTIMAL LINEAR APPROXIMATION
}

\author{
BY WOLFGANG DAHMEN ${ }^{1}$ AND ERNST GORLICH
}

Communicated by M. Golomb, May 28, 1974

In 1964, M. Golomb, in his survey paper on optimal and nearly-optimal linear approximation, presented at the General Motors Conference [3], called attention to an unsolved problem. It is the purpose of this note to solve this problem and at the same time to give a certain extension of the Haršiladze-Lozinskii theorem.

The authors are indebted to Professor P. L. Butzer for many helpful discussions and for a critical reading of the manuscript.

Let $C_{2 \pi}$ be the space of continuous $2 \pi$-periodic functions with Čebyšev norm, $\Pi_{n}$ the class of trigonometric polynomials of degree $\leqslant n$, and $E_{n}[f]=$ $\inf \left\{\|f-p\| ; p \in \Pi_{n}\right\}$ the error of best approximation of an $f \in C_{2 \pi}$ by elements of $\Pi_{n}$ for an $n \in \mathbf{P}=\{0,1,2, \cdots\}$. A sequence $\left\{U_{n}\right\}_{n \in \mathbf{P}}$ of bounded linear operators on $C_{2 \pi}$ into $C_{2 \pi}$ is called asymptotically optimal [3] for a given subset $Y \subset C_{2 \pi}$ if

$$
\sup _{f \in Y}\left\|f-U_{n} f\right\| \leqslant M_{Y} \sup _{f \in Y} E_{n}[f] \quad(n \in \mathbf{P}),
$$

$M_{Y}$ being some constant. $\left\{U_{n}\right\}$ is called optimal for $Y$ if (1) is satisfied with $M_{Y}=1$.

In particular, $Y$ will be taken to be one of the spaces $C_{0}^{r}, r \in \mathbf{P}$ or $A_{0}^{\alpha}, \alpha>0$, where $C_{0}^{r}$ consists of those $f \in C_{2 \pi}$ whose $r$ th derivative is continuous and satisfies $\left\|f^{(r)}\right\| \leqslant 1$, and $A_{0}^{\alpha}$ is the class of functions $f(z)$ of a complex variable $z=x+i y$ which are $2 \pi$-periodic in $x$, real for $y=0$, analytic in the open strip $|y|<\alpha$, continuous in $|y| \leqslant \alpha$, and satisfy

AMS (MOS) subject classifications (1970). Primary 42A08; Secondary 41 A25, 41A35, $41 \mathrm{~A} 50$.

Key words and phrases. Best trigonometric approximation, linear polynomial operators, optimal rate of approximation, Hařsiladze-Lozinskiĭ theorem.

${ }^{1}$ Supported by a DFG research grant (Bu 166/21) which is gratefully acknowledged. 
$\sup _{|y| \leqslant \alpha,|x| \leqslant \pi}|\operatorname{Re} f(z)| \leqslant 1$. By the Jackson and Bernstein theorems, a sequence $\left\{U_{n}\right\}$ of bounded linear operators is asymptotically optimal for come $C_{0}^{r}$ [some $A_{0}^{\alpha}$ ] iff $\left\|f-U_{n} f\right\|=O\left(n^{-r}\right)\left[O\left(e^{-\alpha n}\right)\right], n \rightarrow \infty$, for all $f \in C_{0}^{r}$ $\left[f \in A_{0}^{\alpha}\right]$. Moreover, since $\sup \left\{E_{n}[f] ; f \in C_{0}^{r}\right\}=\mu_{r}(n+1)^{-r}$ for all $n \in$ $\mathbf{P}$, where $\mu_{r}$ denote the Favard-Achieser-Krein constants $(r \in \mathbf{P})$, a sequence $\left\{U_{n}\right\}$ is optimal for some $C_{0}^{r}$ iff $\left\|f-U_{n} f\right\| \leqslant \mu_{r}(n+1)^{-r}$ for all $f \in C_{0}^{r}$, $n \in \mathbf{P}$.

Golomb's conjecture [3] consists of the following two statements.

(A) There does not exist a sequence $\left\{U_{n}\right\}$ of bounded linear polynomial (i.e. $U_{n}\left(C_{2 \pi}\right) \subset \Pi_{n}$ for all $n \in \mathbf{P}$ ) operators which is asymptotically optimal for all the classes $C_{0}^{r}, r \in \mathbf{P}$, and at the same time for all the classes $A_{0}^{\alpha}, \alpha>0$.

(B) There does not exist a sequence of bounded linear polynomial operators which is optimal for all classes $C_{0}^{r}, r \in \mathbf{P}$.

In case (A), this was motivated by the fact that the Fourier partial sums $S_{n}$ are asymptotically optimal for each $A_{0}^{\alpha}, \alpha>0$, but not for any $C_{0}^{r}$, $r \in \mathbf{P}$, whereas the de La Vallée Poussin sums $V_{n}=(n-[n / 2]+1)^{-1}$. $\Sigma_{k=[n / 2]}^{n} S_{k}$ are asymptotically optimal for each $C_{0}^{r}, r \in \mathbf{P}$, but not for any $A_{0}^{\alpha}, \alpha>0$. Concerning (B), for each class $C_{0}^{r}$ there exists an optimal sequence of convolution type operators, but it depends on $r$ and is unique at least among convolutions.

To prove (A) assume the contrary to be valid. If $\left\{U_{n}\right\}$ is the sequence in question, define a sequence $\left\{\bar{U}_{n}\right\}$ of bounded linear polynomial operators by

$$
\bar{U}_{n} f=\frac{1}{2 \pi} \int_{-\pi}^{\pi} T_{-t} U_{n} T_{t} f d t, \quad T_{t} f(x)=f(x+t),
$$

according to Marcinkiewicz' device [5]. Then the $\bar{U}_{n}$ are convolutions and they are asymptotically optimal for all $C_{0}^{r}, A_{0}^{\alpha}, r \in \mathrm{P}, \alpha>0$ as well. Thus the following two theorems may be applied in order to derive a contradiction.

THEOREM 1. If $\left\{U_{n}\right\}$ is a sequence of bounded linear polynomial operators on $C_{2 \pi}$ which is asymptotically optimal for some $A_{0}^{\alpha}, \alpha>0$, then $\lim \sup _{n \rightarrow \infty}\left\|U_{n}\right\|=+\infty$.

THEOREM 2. If $\left\{U_{n}\right\}$ is a sequence of bounded linear polynomial convolution operators on $C_{2 \pi}$ which is asymptotically optimal for some $C_{0}^{r}$, $r \in \mathbf{P}$, then $\left\|U_{n}\right\|=O(1), n \rightarrow \infty$. 
The proof of Theorem 1 proceeds via (2) and makes use of a weak version of an inequality of Hardy-Littlewood [4] and Sidon [8] (to be found e.g. in Nikol'skii [6, p. 262]). Theorem 2 is proved by an application of Bernstein's inequality to $\left(U_{n}-V_{n}\right) f$.

For the proof of (B) assume that $\left\{U_{n}\right\}$ satisfies $\left\|f-U_{n} f\right\| \leqslant$ $\mu_{r}(n+1)^{-r}$ for all $f \in C_{0}^{r}, n, r \in \mathrm{P}$. Then the following Lemma furnishes a contradiction to the fact that the $\mu_{r}$ are bounded uniformly in $r$.

LEMMA. If $\left\{U_{n}\right\}$ is a sequence of bounded linear polynomial operators on $C_{2 \pi}$ such that for each $r \in \mathbf{P}$

$$
\sup _{f \in C_{0}^{r}}\left\|f-U_{n} f\right\| \leqslant M_{r}(n+1)^{-r} \quad\left(f \in C_{0}^{r}, n \in \mathbf{P}\right)
$$

then $\lim \sup _{r \rightarrow \infty} M_{r}=+\infty$.

This is a consequence of (2) and of the inequality mentioned above (see [8]).

In this context let us mention the familiar Haršiladze-Lozinskii theorem (see e.g. [2, pp. 212, 233]) which asserts that there does not exist a sequence $\left\{U_{n}\right\}$ of bounded linear polynomial operators satisfying simultaneously

(a) $U_{n}\left(U_{n} f\right)=U_{n} f$ for each $n \in \mathbf{P}, f \in C_{2 \pi}$, and

(b) $\left\|f-U_{n} f\right\| \rightarrow 0$ as $n \rightarrow \infty$ for each $f \in C_{2 \pi}$.

Extensions of this result have been given e.g. by Berman [1] and Sapogov [7]. As a consequence of the above, another extension is obtained on replacing the projection condition (a) by $\left(\mathrm{a}^{\prime}\right)$ or $\left(\mathrm{a}^{\prime \prime}\right)$ below.

(a') $\left\{U_{n}\right\}$ is asymptotically optimal for some $A_{0}^{\alpha}, \alpha>0$.

(a") $\left\{U_{n}\right\}$ satisfies (3) for each $r \in \mathbf{P}$, and $M_{r}=O(1), r \rightarrow \infty$.

Details will appear elsewhere.

\section{REFERENCES}

1. D. L. Berman, On the impossibility of constructing a linear polynomial operator furnishing an approximation of the order of best approximation, Dokl. Akad. Nauk SSSR 120 (1958), 1175-1177. (Russian) MR 20 \#5387.

2. E. W. Cheney, Introduction to approximation theory, McGraw-Hill, New York, 1966. MR 36 \#5568;

3. M. Golomb, Optimal and nearly-optimal linear approximations, Approximation of Functions (ed. by H. L. Garabedian), Elsevier, Amsterdam, 1965, pp. 83-100. MR 34 \#1767.

4. G. H. Hardy and J. E. Littlewood, Some new properties of Fourier-constants, Math. Ann. 97 (1927), 158-208. 
5. J. Marcinkiewicz, Quelques remarques sur l'interpolation, Acta Litt. Acad. Sci. Szeged 8 (1937), 127-130.

6. S. M. Nikol'skir, On linear methods of summation of Fourier series, Izv. Akad. Nauk SSSR Ser. Mat. 12 (1948), 259-278. (Russian) MR 10, 247.

7. N. A. Sapogov, Norms of linear polynomial operators, Dokl. Akad. Nauk SSSR 143 (1962), 1286-1288 = Soviet Math. Dokl. 3 (1962), 602-604. MR 25 \#381b.

8. S. Sidon, Über Fourier-Koeffizienten, J. London Math. Soc. 13 (1938), 181-183.

LEHRSTUHL A FÜR MATHEMATIK, TECHNOLOGICAL UNIVERSITY, 51 AACHEN, FEDERAL REPUBLIC OF GERMANY 